International Journal on Soft Computing (IJSC) Vol.6, No. 2, May 2015

\title{
FUZZY ENTROPY BASED OPTIMAL THRESHOLDING TECHNIQUE FOR IMAGE ENHANCEMENT
}

\author{
U.Sesadri ${ }^{1}$,B. Siva Sankar ${ }^{2}$, C. Nagaraju ${ }^{3}$ \\ Assistant.Professor\&Head of CSE, Vaagdevi Institute of Technology and Science, \\ Proddature $^{1}$ \\ M.Tech student of Vaagdevi Institute of Technology and Science, Proddatur ${ }^{2}$ \\ Assoc. Professor\&Head of CSE, YSRCE of YVU, Proddatur ${ }^{3}$
}

\begin{abstract}
Soft computing is likely to play aprogressively important role in many applications including image enhancement. The paradigm for soft computing is the human mind. The soft computing critique has been particularly strong with fuzzy logic. The fuzzy logic is facts representationas a rule for management of uncertainty. Inthis paperthe Multi-Dimensional optimized problem is addressed by discussing the optimal thresholding usingfuzzyentropyfor Image enhancement. This technique is compared with bi-level and multi-level thresholding and obtained optimal thresholding values for different levels of speckle noisy and low contrasted images. The fuzzy entropy method has produced better results compared to bi-level and multi-level thresholding techniques.
\end{abstract}

\section{KEY WORDS}

fuzzy entropy, segmentation, soft computing, MAD and optimal thresholding

\section{INTRODUCTION}

Soft computing approaches have been applied to numerous real-world problems. Applications can be found in image segmentation, pattern recognition, image enhancement and industrial inspection, speech processing, robotics, naturallanguage understanding, etc. Thethresholding technique is a reckless, modern and computationally low-cost segmentation technique that is always serious and decisive in image enhancementuses. The value of image thresholding is not always satisfactory because of the presence of noise and vagueness and ambiguity among the classes. In [1], a three-level thresholding method has been presented for image enhancement based on probability partition, fuzzy partition and entropy concept. This concept can be easily extended to $\mathrm{N}$-level $(\mathrm{N}>3)$ thresholding. However it fails for multi-resolution real images. In [2] the image thresholding by using cluster organization from the histogram of an imageisproposed based on inter-class variance of the clusters to be merged and the intra-class variance of the new merged cluster. In [3], multi-level thresholding technique is presented for color image segmentation by maximizing the conditional entropy but Fuzziness in images due to noise poses a 
great challengein image segmentation and thresholding.In future the abovemethod may be comprehensive to deal with noisy images by use offuzzy tools etc. In [4] this paper presents a new histogram thresholding methodology using fuzzy and rough set theories. The power of the proposed methodology lies in the fact that it does not make any prior assumptions about the histogram unlike many existing techniques.In [5], fuzzy c means threshold clustering method was used for underwater images. This emphasizesthe necessity of image segmentation, which splits an image intoparts that take strong correlations with objects to reflect theactual information collected from the real world when compare tooriginal image fuzzy method gives cut down value. In [6] Unsupervised Image Thresholding using Fuzzy Measures is presented. This method successfully segmenting the images ofbimodal and multi-model histograms for the automatic selection of seedsubsets to decide the effective ROI. In [7], Bacterial Foraging (BF) algorithm based on Tsallis objective function is presented for multilevel thresholding in image segmentation. In [8], two-stage fuzzy set theoretic approach to image thresholding that uses the measure of fuzziness to evaluate the fuzziness of an image and to find an optimal threshold value is proposed. But this method is not appropriate to color images. In [9], fast image segmentation methods based on swarm intelligence and 2-D Fisher criteria thresholding were used for image segmentation. In [10], was used a procedure like thresholding by fuzzy c-means (THFCM) algorithm for image segmentation to find an automatic threshold value. In [11], An Automatic Multilevel Thresholding Method for Image segmentation was proposed based on Discrete Wavelet Transforms and Genetic Algorithm. It worksonly for synthetic and real images. In [12] used thresholding technique with genetic algorithm to find optimal thresholds between the various objects and the background. In [13], an image segmentation framework which applied automatic thresholding selection by means of fuzzy set theory and density model. In [14],Otsu andcanny edge detectionswere the two techniques used for image segmentation. In [15], image segmentation was implemented based on thresholding on Gaussian and salt \& pepper noises. It is not appropriate for other noises.

\section{BI-LEVEL THRESHOLDING}

The below gray-levelbimodal histogram shown in Fig 1) corresponds to an image, f1(x,y), composed of objects overlapping with background of theimage. One exact way to extract the objects from the background image is to select a threshold 'Th' that separates the modes. And then any point $(\mathrm{x}, \mathrm{y})$ for which $\mathrm{f} 1(\mathrm{x}, \mathrm{y})>\mathrm{Th}$, is called an object point; if not, the point is called a background point.

In given image, let the gray levels are $\mathrm{L}$ and the range is from $\{0,1,2 \ldots(\mathrm{L}-1)\}$. Then the gray level occurrence probability $\mathrm{k}$ is defined by following equation:

$$
p_{r k}=\frac{h(k)}{N} \text { for }(0 \leq k \leq(L-1))
$$

Where $h(k)$ is corresponding gray level number of pixels, $\mathrm{N}$ is the image total number of pixels that is equal to $\sum_{i=0}^{L-1} h(i)$.

The thresholding problem of bi-level can be described as follows

$$
\operatorname{MaximizeJ}(t)=H E_{0}+H E_{1}
$$


Where

$$
\begin{aligned}
H E_{0}=-\sum_{i=0}^{t-1} \frac{p r_{i}}{w_{0}} \ln \frac{p r_{i}}{w_{0}}, & w_{0}=\sum_{i=0}^{t-1} p r_{i} \text { and } \\
H E_{1}=-\sum_{i=0}^{L-1} \frac{p r_{i}}{w_{1}} \ln \frac{p r_{i}}{w_{1}}, & w_{1}=\sum_{i=0}^{L-1} p r_{i} \text { and }
\end{aligned}
$$

And the eq. (1) maximizes optimal threshold in the gray level.

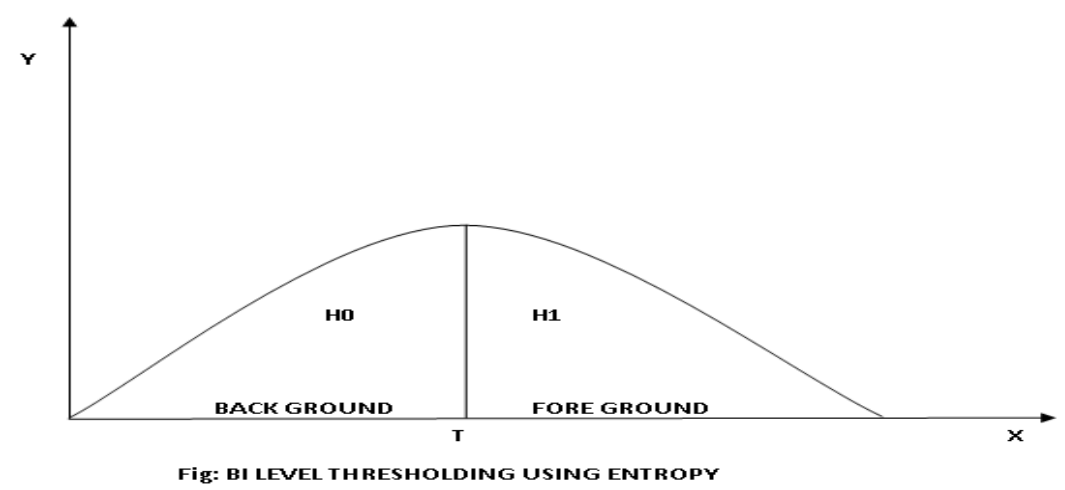

Fig1

This method produced better results for there the object and background pixels have gray levels and grouped into two dominant modes.However bi-level thresholding technique fails for overlapping image objects with background.

\section{MULTILEVEL THRESHOLDING}

The more general case of bi-level thresholding technique, where the multiple overlapping objects characterizes the image histogram.Fig (2) shows thatuni-modelmultiple thresholding classifies a point $(\mathrm{x}, \mathrm{y})$ as belonging to the one object class in $\operatorname{Th} 1<\mathrm{f} 1(\mathrm{x}, \mathrm{y}) \leq \mathrm{Th} 2$, to the other object class if $\mathrm{f} 1(\mathrm{x}, \mathrm{y})>\mathrm{Th} 2$, and to the background if $\mathrm{f} 1(\mathrm{x}, \mathrm{y}) \leq \mathrm{Th} 1$. The segmentation problems require multiple thresholds for the quality enhancement. The multilevel threshold is the extension to the Kapur's entropy criterion and this method is treating as the optimal multilevel thresholding problem.In the given image [th1, th2, th $3 \ldots \mathrm{th}_{\mathrm{m}}$ ] the optimal threshold is calculated as follows and it maximizes the following objective function:

$$
J([t h 1, t h 2, \ldots ., t h m])=H E_{0}+H E_{1}+H E_{2}+\cdots+H E_{m}
$$

Where

$$
H E_{0}=-\sum_{i=0}^{t h 1-1} \frac{p r_{i}}{w_{0}} \ln \frac{p r_{i}}{w_{0}}, \quad w_{0}=\sum_{i=0}^{t h 1-1} p r_{i}
$$


International Journal on Soft Computing (IJSC) Vol.6, No. 2, May 2015

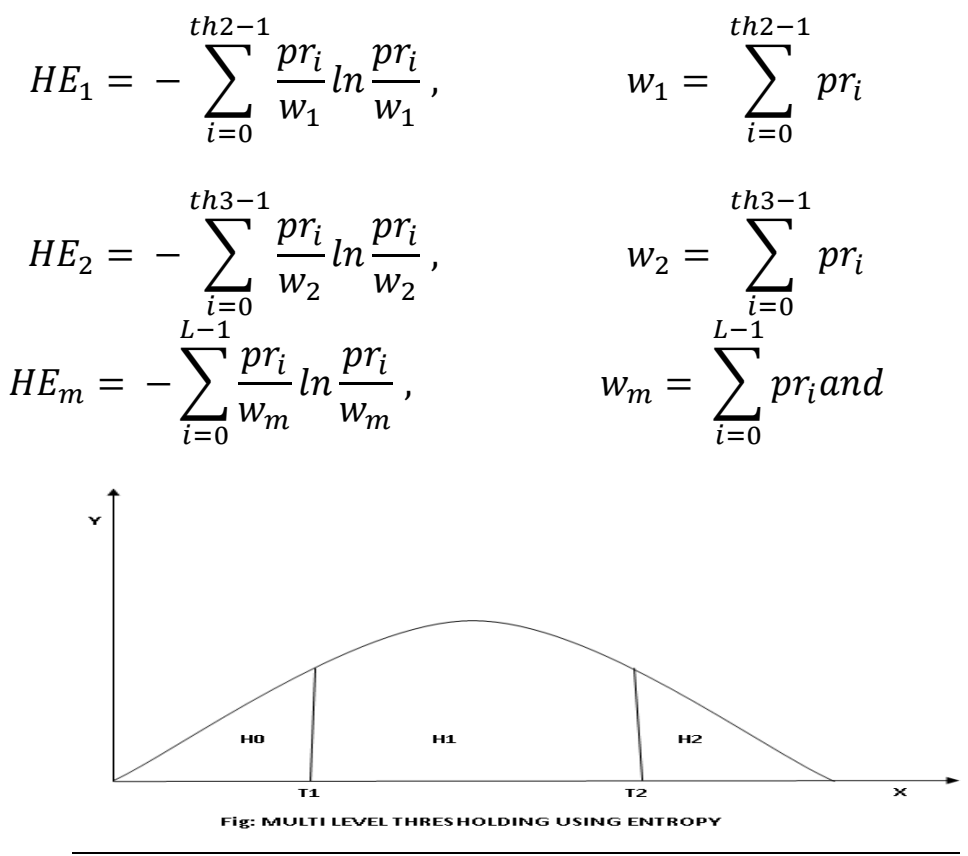

Fig 2

This method also works for overlapping images by considering multiple threshold values but however it is not suitable for low contrasted images.

\section{FUZZY ENTROPY TECHNIQUE}

The fuzzy entropy is a statistical measure of randomness in animage, the pixel values, and Dmi occur with probabilitiespr(Dmi), which are given by the bin heights of the normalizedhistogram. The maximum fuzzy entropy principle based on probability partition is defined as Let $\mathrm{Dm}=\{$ $(\mathrm{i}, \mathrm{j}): \mathrm{i}=0,1, \ldots \mathrm{M}-1 ; \mathrm{j}=0,1, . \mathrm{N}-1\}, \mathrm{Gr}=\{0,1 \ldots \mathrm{l}-1\}$, where $\mathrm{M}, \mathrm{N}$ and 1 are 3 positive integers. Thus a digitized picture defines a mapI:Dm $\rightarrow G r$. Let $\operatorname{In}(\mathrm{x}, \mathrm{y})$ be the gray level value of the image at the pixel $(\mathrm{x}, \mathrm{y})$

$$
\begin{gathered}
\operatorname{In}(x, y) \in \operatorname{Gr} \forall(x, y) \in D m \\
D m_{k}=\{(x, y): \operatorname{In}(x, y)=k,(x, y) \in D m \\
\mathrm{K}=0,1,2 \ldots \mathrm{M}-1 \\
h_{k}=\frac{n_{k}}{N * M}, \quad k=0,1, \ldots ., m-1
\end{gathered}
$$

Where $n_{k}$ denotes the number of pixels in $D m_{k}$. The following conclusions can be easily formed:

$$
\begin{aligned}
& \bigcup_{k=0}^{m-1} D m_{k}=D m, \quad D m_{j} \cap D m_{k}=\varnothing(k \neq j) \\
& 0 \leq h_{k} \leq 1, \quad \sum_{k=0}^{m-1} h_{k}=1, \quad k=0,1, \ldots . m-1
\end{aligned}
$$


International Journal on Soft Computing (IJSC) Vol.6, No. 2, May 2015

$$
\begin{aligned}
& p r_{k d}=p r\left(D m_{k d}\right)=p r_{k} * p r_{d / k} \\
& p r_{k m}=p r\left(D m_{k m}\right)=p r_{k} * p r m / k \\
& p r_{k b}=p r\left(D m_{k b}\right)=p r_{k} * p r_{b} / k \\
& p r_{d / k}+p r m_{k}+p r_{b / k}=1 \\
& 1 \quad\left(\begin{array}{cl}
1 & k
\end{array}\right. \\
& 1-\frac{(k-p 1)^{2}}{(r 1-p 1) *(q 1-p 1)} p 1<k \leq q 1 \\
& \frac{(k-r 1)^{2}}{(r 1-p 1) *(r 1-q 1)} q 1<k \leq r 1 \\
& 0 \\
& k>r 1 \\
& \Psi_{m}(k)=\left\{\begin{array}{ccc}
0 & k \leq p 1 \\
& \frac{(k-p 1)^{2}}{(r 1-p 1) *(q 1-p 1)} p 1<k \leq q 1 \\
& 1-\frac{(k-r 1)^{2}}{(r 1-p 1) *(r 1-q 1)} q 1<k \leq r 1 \\
1 & 1-\frac{(k-p 2)^{2}}{(r 2-p 2) *(q 2-p 2)} p 2<k \leq q 2 \\
& \frac{(k-r 2)^{2}}{(r 2-p 2) *(r 2-q 2)} q 2<k \leq r 2 & k 1<k \leq p 2 \\
& \quad & k>r 2
\end{array}\right. \\
& \Psi_{b}(k)=\left\{\begin{array}{cc}
0 & k \leq p 2 \\
\frac{(k-p 2)^{2}}{(r 2-p 2) *(q 2-p 2)} p 2<k \leq q 2 \\
1-\frac{(k-r 2)^{2}}{(r 2-p 2) *(r 2-q 2)} q 2<k \leq r 2 \\
1 \quad & k>r 2
\end{array}\right.
\end{aligned}
$$

Where six parameters $\mathrm{p} 1, \mathrm{q} 1, \mathrm{r} 1, \mathrm{p} 2, \mathrm{q} 2, \mathrm{r} 2$ satisfy the following condition:

$$
0<p 1 \leq q 1 \leq r 1 \leq p 2 \leq q 2 \leq r 2<255
$$

The fuzzy entropy function of every class is specifiedunder:

$$
\begin{aligned}
H E_{d} & =-\sum_{k=0}^{255} \frac{p r_{k} * \Psi_{d}(k)}{p r_{d}} * \ln \left\{\frac{p r_{k} * \Psi_{d}(k)}{p r_{d}}\right\} \\
H E_{m} & =-\sum_{k=0}^{255} \frac{p r_{k} * \Psi_{m}(k)}{p r_{m}} * \ln \left\{\frac{p r_{k} * \Psi_{m}(k)}{p r_{m}}\right\}
\end{aligned}
$$


International Journal on Soft Computing (IJSC) Vol.6, No. 2, May 2015

$$
H E_{b}=-\sum_{k=0}^{255} \frac{p r_{k} * \Psi_{b}(k)}{p r_{b}} * \ln \left\{\frac{p r_{k} * \Psi_{b}(k)}{p r_{b}}\right\}
$$

Then the sum fuzzy entropy function is specified as follow:

$$
H E(p 1, q 1, r 1, p 2, q 2, r 2)=H E_{d}+H E_{m}+H E_{b}
$$

Along with six variables $\mathrm{p} 1, \mathrm{q} 1, \mathrm{r} 1, \mathrm{p} 2, \mathrm{q} 2, \mathrm{r} 2$ the total fuzzy entropy is varied. An optimal combination of (p1, q1, r1, p2, q2, r2) can be found.So that the total fuzzy entropy HE (p1, q1, r1, $\mathrm{p} 2, \mathrm{q} 2, \mathrm{r} 2$ ) has the maximum value.

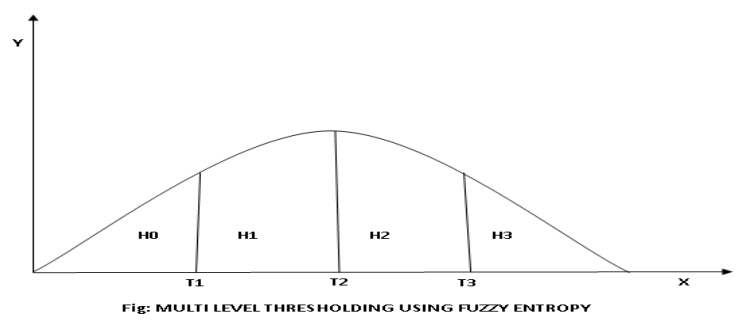

Fig 3

\section{QUALITY PARAMETERS}

5.1. Mean:mean is defined in terms of theaverage gray levels of the imagewith human observations. In gray image the mean is calculated as

$$
\operatorname{Mean}(\mu)=\frac{1}{P Q} \sum_{x=1}^{P} \sum_{y=1}^{Q} g 1(x, y)
$$

Where $\mathrm{P}, \mathrm{Q}$ are the width and height in terms of the gray level pixels the image and $\mathrm{g} 1(\mathrm{x}, \mathrm{y})$ is gray value.

5.2. Standard deviation: the standard deviation of gray level image iscalculated as follows

$$
S t(\sigma)=\sqrt{\frac{1}{P Q} \sum_{x=1}^{P} \sum_{y=1}^{Q}(g 1(x, y)-\mu)^{2}}
$$

Where $\mathrm{P}, \mathrm{Q}$ are the width and height of the image, $\mu$ is mean of the image, $\mathrm{g} 1(\mathrm{x}, \mathrm{y})$ is gray level value of the image, $S t(\sigma)$ is standard deviation

5.3. Mean Absolute deviation: The gray levels Mean Absolute deviation (MAD) from the median is calculated as follows

$$
M A D=\frac{1}{P Q} \sum_{x=1}^{P} \sum_{y=1}^{Q} \mid g 1(x, y)-\text { median } \mid
$$

Where $\mathrm{M}, \mathrm{N}$ are the width and height of the image in terms of pixels, median is the median gray value of the image mask.

5.4. The Manhattan distance function computes the distance that would be traveled to get from one data point to theother point. The Manhattan distance between two images is the sum of the differences of their corresponding components.

Manhattan Distance $=$ Abs $(\mathrm{a}-\mathrm{x})+$ Abs $(\mathrm{b}-\mathrm{y})+\operatorname{Abs}(\mathrm{c}-\mathrm{z})$ 
Where $(\mathrm{a}, \mathrm{b}, \mathrm{c})$ and $(\mathrm{x}, \mathrm{y}, \mathrm{z})$ are two referenced points to bematched.

\section{EXPERIMENTAL RESULTS}

In this paper, we have presented a new gray level thresholdingalgorithm based on the close relationship betweenthe image thresholding problem and the fuzzylogic. In order to evaluate the performance of the proposed fuzzy entropy technique, it has been tested using imageswith low contrast andspeckle noise.The parameters like mean, standard deviation, MAD and Manhattan distance function are calculated and results are shown in the form tables and graphs. Table1 to table3 show the bi-level, multi-level and fuzzy entropy technique's mean standard deviation, mean-absolute-deviation and Manhattan distance function values respectively. The fuzzy entropy technique is applied on low contrasted name plate with altered noise levels. Fuzzy entropy technique is better for low contrasted name plate with noise up to $65 \%$ of speckle noise. From the evaluation of the resultant images, were solved that the fuzzy entropy thresholding technique yields betterimages than those obtained by the widely used Bi-level thresholding and multi-level thresholding methods.

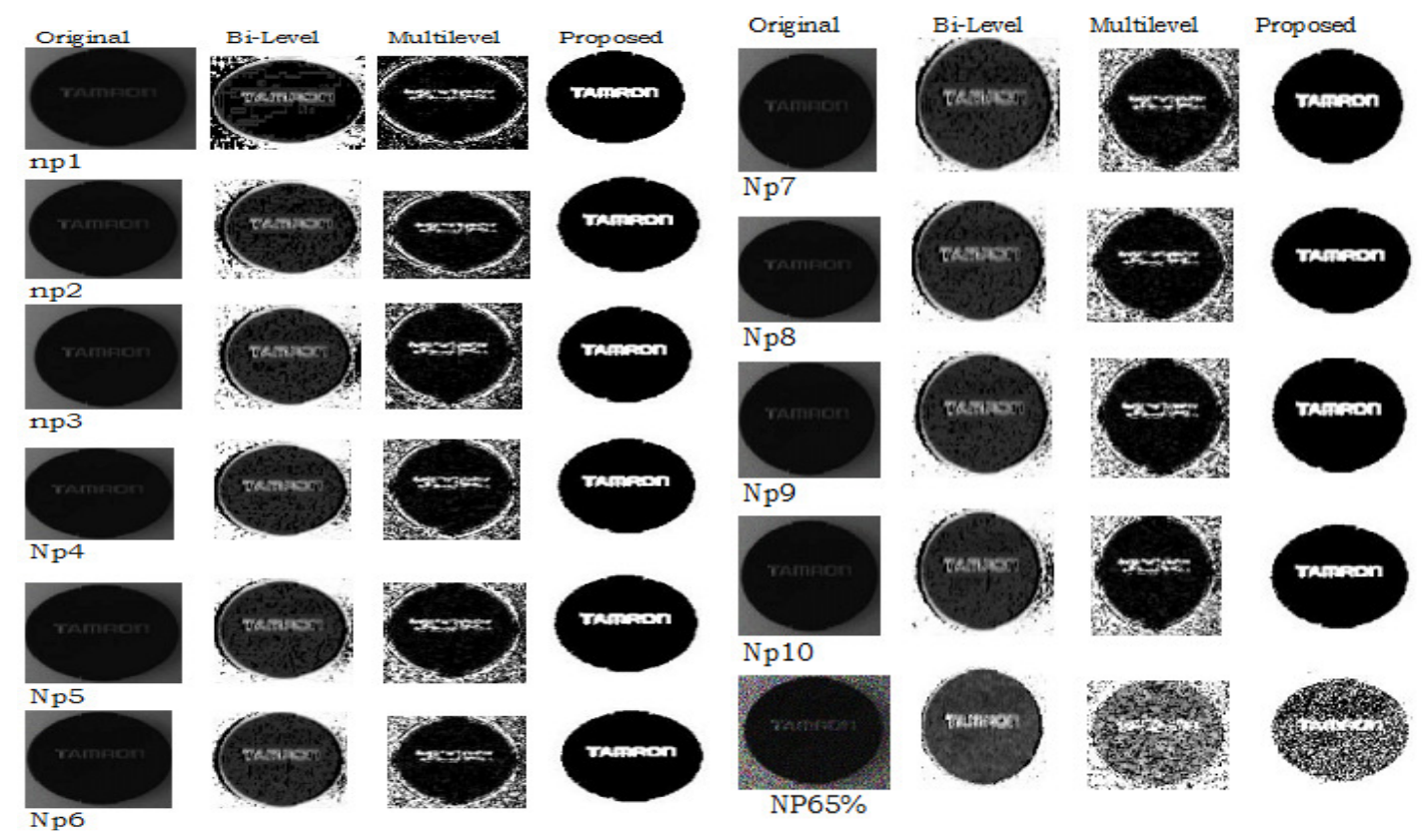

Fig 4: Comparison of Three Methods with Speckle Noise 
International Journal on Soft Computing (IJSC) Vol.6, No. 2, May 2015

\begin{tabular}{|c|c|c|c|c|c|c|c|c|c|c|c|c|c|c|}
\hline Images & Mean & SD & MAD & MDF & images & mean & SD & MAD & MDF & Image & Mean & SD & MAD & MDF \\
\hline NP1 & 0.1886 & 0.1553 & 0.8112 & 0.1888 & NP1 & 0.0622 & 0.0855 & 0.8464 & 0.1536 & NP1 & 0.0444 & 0.0301 & 0.9990 & 0.0010 \\
\hline NP2 & 0.2180 & 0.1504 & 0.7877 & 0.2123 & NP2 & 0.1041 & 0.0976 & 0.8063 & 0.1937 & NP2 & 0.1778 & 0.0296 & 0.9990 & 0.0010 \\
\hline NP3 & 0.2261 & 0.1475 & 0.7824 & 0.2176 & NP3 & 0.1401 & 0.1017 & 0.7725 & 0.2275 & NP3 & 0.0889 & 0.0294 & 0.9990 & 0.0010 \\
\hline NP4 & 0.2330 & 0.1488 & 0.7772 & 0.2228 & NP4 & 0.1684 & 0.1005 & 0.7504 & 0.2496 & NP4 & 0.1333 & 0.0301 & 0.9990 & 0.0010 \\
\hline NP5 & 0.2377 & 0.1476 & 0.7727 & 0.2273 & NP5 & 0.1921 & 0.0996 & 0.7305 & 0.2695 & NP5 & 0.2667 & 0.0314 & 0.9988 & 0.0012 \\
\hline NP6 & 0.2453 & 0.1464 & 0.7709 & 0.2291 & NP6 & 0.2097 & 0.0999 & 0.7156 & 0.2844 & NP6 & 0.3556 & 0.0339 & 0.9987 & 0.0013 \\
\hline NP7 & 0.2473 & 0.1454 & 0.7691 & 0.2309 & NP7 & 0.2291 & 0.0986 & 0.7031 & 0.2969 & NP7 & 0.4889 & 0.0336 & 0.9986 & 0.0014 \\
\hline NP8 & 0.2513 & 0.1463 & 0.7668 & 0.2332 & NP8 & 0.2304 & 0.0974 & 0.6992 & 0.3008 & NP8 & 0.3556 & 0.0340 & 0.9987 & 0.0013 \\
\hline NP9 & 0.2503 & 0.1460 & 0.7670 & 0.2330 & NP9 & 0.2515 & 0.0992 & 0.6832 & 0.3168 & NP9 & 0.0000 & 0.0374 & 0.9982 & 0.0018 \\
\hline NP10 & 0.2549 & 0.1444 & 0.7643 & 0.2357 & NP10 & 0.2549 & 0.0993 & 0.6803 & 0.3197 & NP10 & 0.2222 & 0.0357 & 0.9984 & 0.0016 \\
\hline \multicolumn{5}{|c|}{ Table1: bi-level Thresholding } & & Table2: & Ilti-level & Threshol & ding & \multicolumn{5}{|c|}{ Table 3: Fuzzy Entropy Thresholding } \\
\hline
\end{tabular}

Tabe1: bi-level thresholdingTable2: Multi-level thresholdingTable3: fuzzy entropy thresholding

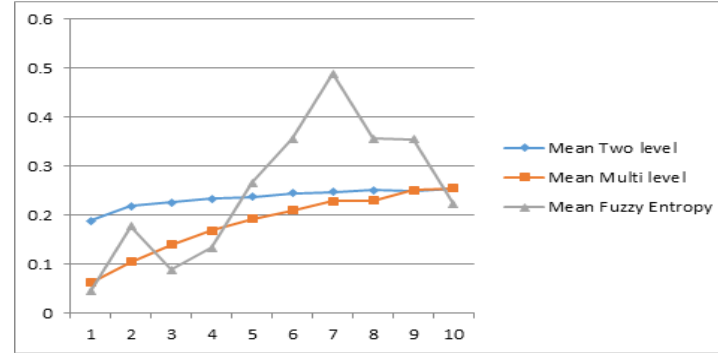

Graph1: Mean

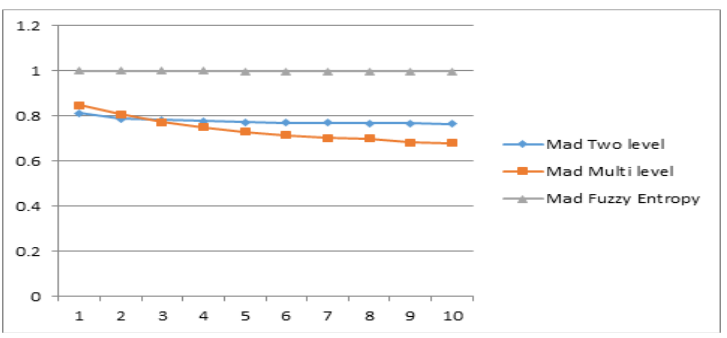

Graph3: mean absolute deviationGraph

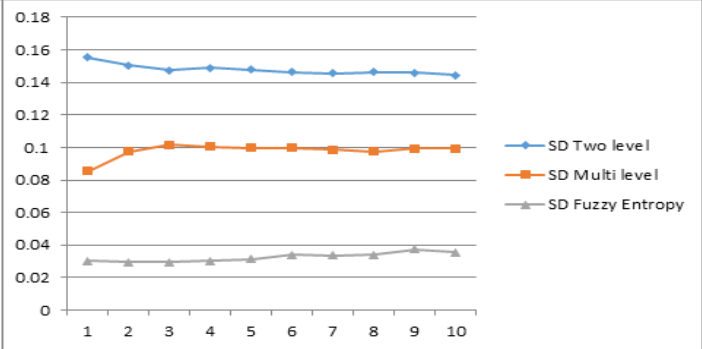

Graph2: standard deviation

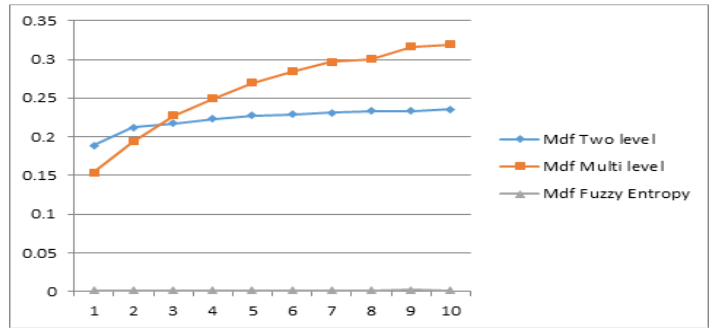

4: Manhattan distance

\section{CONCLUSION}

In this paper the optimal threshold values are determined by three thresholding techniques of bilevel, multi-level and fuzzy entropy and they are tested with a variety of representing low contrasted as well as natural images with noise for Image enhancement. In proposed method, fuzzy entropy thresholding is used for image enhancement and compared results with existing bilevel and multi-level thresholding methods and proved that proposed method best fit for low contrasted with speckle noise images. The tables and graphs are constructed by standard deviation, mean, MAD and Manhattan distance function. Experiments on low contracted, noisy and real images have proved the robustness of the proposed technique. Yet the fuzzy entropy technique is not appropriate for salt \& pepper and Gaussian noises. 
International Journal on Soft Computing (IJSC) Vol.6, No. 2, May 2015

\section{REFERENCES}

[1]Wen-Bing Tao, Jin-Wen Tian, Jian Liu, "Image segmentation by three-level thresholding based on maximum fuzzy entropy and genetic algorithm" 2003 Pattern Recognition Letters 24, pp. 3069-3078

[2]AgusZainalArifin, Akira Asano, "Image segmentation by histogram thresholdingusing hierarchical cluster analysis" 2006 Pattern Recognition Letters xxx, pp. xxx-xxx

[3]R.Sukesh Kumar, Abhisek Verma and Jasprit Singh, "Color Image Segmentation and Multi-Level Thresholding by Maximization of Conditional Entropy" 2007 World Academy of Science, Engineering and Technology on International Journal of Computer, Control, Quantum and Information Engineering, Vol: 1, No: 6, pp. 1607-1615

[4]Debashis Sen and Sankar K. Pal, "Histogram Thresholding Using Fuzzy and Rough Measures of Association Error" 2009 ieee transactions on image processing, VOL. 18, NO. 4, pp. 879-889

[5]Dr.G.Padmavathi, Mr.M.Muthukumar and Mr. Suresh Kumar Thakur, "Nonlinear Image segmentation using fuzzy c means clustering method with thresholding for underwater images" 2010 IJCSI International Journal of Computer Science Issues, Vol. 7, Issue 3, No 9,pp. 35-40

[6]M Seetharama Prasad, T Divakar, B Srinivasa Rao and Dr C Naga Raju, "Unsupervised Image Thresholding using Fuzzy Measures" 2011 International Journal of Computer Applications (0975 8887), Volume 27- No.2, pp. 32-41

[7]P.D. Sathya, and R. Kayalvizhi, "modified bacterial foraging algorithm based multi-level thresholding for image segmentation” 2011 Engineering Applications of Artificial Intelligence 24, pp. 595-615

[8]Ambar Dutta, AvijitKar and B. N. Chatterji, "fuzzy set theoretic approach to image thresholding" 2011 International Journal of Computer Science, Engineering and Applications (IJCSEA) Vol.1, No.6,pp.63-72

[9]Zhiwei Ye, Zhengbing Hu, Xudong Lai and Hongwei Chen, "Image Segmentation Using Thresholding and Swarm Intelligence" 2012 journal of software, VOL. 7, NO. 5, pp. 1074-1082

[10]Firas A. Jassim, "Hybrid Image Segmentation using Discerner Cluster in FCM and Histogram Thresholding" 2012 International Journal of Graphics \& Image Processing IVol 2lissue 4l, pp. 241244

[11]RakeshKumar, TapeshParashar,Gopal Verma, "a multilevel automatic thresholding for image segmentation using genetic algorithm and dwt"International Journal of Electronics and Computer Science Engineering, ISSN- 2277-1956/V1N1-153-160, pp. 153-160

[12]S. Vijayakumar,A.obulasu, Dr.L.S.S. Reddy and C. Nagaraju "reconstruction of digital images by combining Multiple gradients" (C) 2005 - 2009,Journal of Theoretical and Applied Information Technology,vol:10 pp.no.29-34

[13]Jianli Li, Bingbin Dai, Kai Xiao, and Aboul Ella Hassanien, "Density Based Fuzzy Thresholding for Image Segmentation" 2012 A. Ell Hassanien et al. (Eds.): AMLTA 2012, CCIS 322, pp. 118-127

[14]Jamil A. M. Saif, Ali Abdo Mohammed Al-Kubati, AbdultawabSaifHazaa, Mohammed Al- Moraish, "Image Segmentation Using Edge Detection and Thresholding" 2013 The 13th International Arab Conference on Information Technology ACIT, ISSN : 1812-0857, PP.473-476

[15]S.BanuChitra, V.Gayathri and M.Chanda Mona, "a study of image segmentation using thresholding technique on a noisy image" 2014 International Journal of Human Computer Interaction (IJHCI) Singaporean Journal of Scientific Research (SJSR) Vol.6.No.6 2014 Pp. 
International Journal on Soft Computing (IJSC) Vol.6, No. 2, May 2015

\section{AUTHORS}

U. Sesadri is currently working as Assistant. Professor and HOD in the Department of CSE in Vaagdevi Institute of Technology \& science, Proddatur, KadapaDistrict,Andhra Pradesh, India. He received his M.Sc. in Mathematics from SV University, Tirupati, M.E in Computer Science and Engineering from Sathyabama University, Chennai and pursing $\mathrm{PhD}$ in Digital Image Processing from V.T. University, Belgaum. He got 7 years of teaching experience. He attended five National Level workshops and two international level conferences. He organized 10 National levelworkshops and two National level paper presentations.

Bodicherla Siva Sankar is doing his M. tech in Vaagdevi Institute of Technology, Proddatur, Kadapa, A.P, India. He received his B.Tech degree in Information Technology from J.N.T. University Anantapur and he has attended two workshops on cloud computing $\&$ Big Data and Research challenges in Digital Image Processing.

Dr. C. Naga Raju is currently working as Associate Professor and Head of the Department of Computer Science and Engineering at YSR Engineering College of Yogivemana University, Proddatur, Kadapa District, and Andhra Pradesh, India. He received his B.Tech Degree in Computer Science and Engineering from J.N.T.University, Anantapur, and M.Tech Degree in Computer Science from J.N.T.University Hyderabad and $\mathrm{PhD}$ in digital Image processing from J.N.T.University Hyderabad. He has got 18

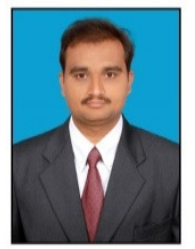
years of teaching experience. He received research excellence award, teaching excellence award and Rayalaseemavidhyaratna award for his credit. He wrote text book on C \& Data structures and Pattern Recognition. He has six PhD scholars. He has published fifty six research papers in various National and International Journals and about thirty research papers in various National and International Conferences. $\mathrm{He}$ has attended twenty seminars and workshops. He delivered 10 keynote addresses. He is member of various professional societies like IEEE, ISTE and CSI. 